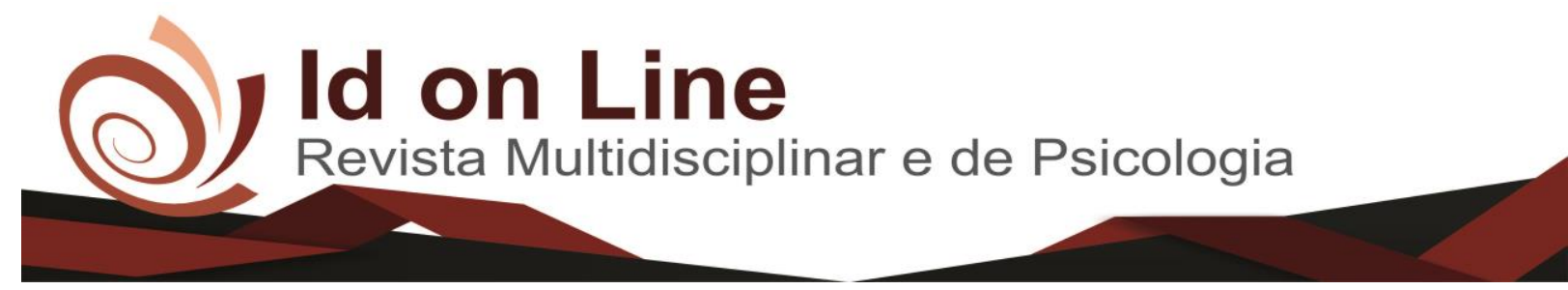

Artigo

\title{
Nível de Informação da Equipe de Enfermagem sobre Segurança do Paciente num Hospital Público
}

Laricy Silva dos Santos ${ }^{1}$; Giovana Fernandes Araújo ${ }^{2}$

\begin{abstract}
Resumo: Objetivou-se compreender o nível de informação da equipe de enfermagem sobre segurança do paciente num hospital público. Trata-se de um estudo exploratório-descritivo de natureza qualitativa. A pesquisa foi realizada em um hospital público, de grande porte, localizado no Sudoeste da Bahia no período de agosto de 2017 através de entrevista gravada utilizando-se um roteiro semiestruturado elaborado e aplicado pelas pesquisadoras. Participaram do estudo 12 enfermeiros que trabalham nas clínicas médica e cirúrgica. As entrevistas foram realizadas individualmente em horário previamente determinado junto aos participantes. Após serem realizadas, as entrevistas foram transcritas para o programa Word/Starter 2010. Os dados foram analisados à luz do referencial de Bardin. Os resultados foram agrupados em três eixos temáticos. Concluiu-se que os profissionais apresentam conhecimento adequado, quando definem segurança do paciente. Foi recorrente a percepção de erros relacionados à medicação também percebeu-se a necessidade de capacitações e treinamentos periódicos para segurança do paciente.
\end{abstract}

Palavras -Chave: Segurança do paciente; Erros; Equipe de Enfermagem.

\section{Information level of Nurse Staff Concerning a Patient's Safety at a Public Hospital}

\begin{abstract}
This study aimed to understand the level of information of the nursing staff about patient's safety in a public hospital. This is an exploratory-descriptive qualitative study. The survey was conducted in a public hospital located in the Southwest of Bahia from August 2017 through recorded interviews using a semi-structured developed and implemented by the researchers. Study participants were 12 nurses working in the medical and surgical clinics. The interviews were conducted individually in predetermined time with the participants. After being made, the interviews were transcribed for the Word program / Starter 2010. The data were analyzed according to the Bardin referential. The results were grouped into three thematic axes. It was concluded that the professionals have adequate knowledge when defining patient safety. It was recurrent the perception of errors related to medication also perceived the need for training and periodic training for patient safety.
\end{abstract}

Keywords: Patient safety; Errors; Nursing staff.

\footnotetext{
${ }^{1}$ Graduanda em enfermagem, pela Faculdade Independente do Nordeste. laricy.silva@ uol.com.br

${ }^{2}$ Enfermeira. Mestre em meio ambiente e sustentabilidade. Docente da Faculdade Independente do Nordeste. giovanafaraujo@hotmail.com
} 


\section{Introdução}

Segurança do paciente compreende um conjunto de medidas adotadas pelos profissionais de saúde, cuja finalidade é evitar danos ao paciente, preservando sua integridade física e moral. Segurança do paciente já era pensada há diversos séculos atrás por Hipócrates, o "pai da medicina", que trazia conceitos de primeiro não causar danos ao indivíduo na prestação de cuidados (BRASIL, 2014). A partir do ano 2000, a segurança do paciente entra como pauta de pesquisas e torna-se internacionalmente valorizada ao ganhar força a constatação de tratar-se de um indicador de qualidade da assistência à saúde (REIS et al, 2013).

Para a Organização Mundial de Saúde (OMS), um em cada dez pacientes pode ser vítima de erros ou eventos adversos advindos da prestação de assistência à saúde (WHO, 2005). Muitas vezes estes erros são noticiados pela mídia, após denúncias de vítimas ou parentes, de forma repetida, principalmente os cometidos pela equipe de enfermagem, gerando um impacto negativo na população ao colocar em dúvida a qualidade dos serviços de saúde. Dentre os erros mais cometidos pelas equipes de saúde, os que envolvem medicação são os que causam maiores danos aos pacientes e estes podem estar presentes em qualquer momento da assistência, como na prescrição, preparo, armazenamento e administração dos medicamentos (LOMBARDI et al, 2016).

Existe um movimento global, que visa a melhoria da segurança do paciente, e consequente melhoria da qualidade da assistência à saúde. Através deste movimento, tem-se realizados esforços, em todos os níveis de atenção à saúde, para proporcionar gerenciamento e minimização de riscos, formação de ambiente seguro, administração segura e correta de medicamentos, dentre outras práticas (DIAS et al, 2014).

Os danos causados aos usuários do sistema de saúde, relacionados aos eventos adversos, provocados por profissionais de saúde, podem ser reduzidos através de medidas como a implantação e utilização de protocolos de segurança do paciente (OLIVEIRA et al, 2014). Os protocolos surgiram a partir da criação, pelo Ministério da Saúde (MS), em 2013, do Programa Nacional de Segurança do Paciente que tem por objetivo prevenir e reduzir a incidência de eventos que gerem danos ao paciente. Os protocolos básicos de segurança são de prática de higiene das mãos; cirurgia segura; segurança durante a prescrição, uso e administração de medicamentos; prevenção de quedas, prevenção do surgimento de úlceras por pressão e identificação do paciente (BRASIL, 2014).

Isto posto, este estudo teve por objetivo: compreender o nível de informação da equipe de enfermagem sobre segurança do paciente num hospital público do sudoeste da Bahia.

Esta pesquisa justifica-se por abordar um tema atual e relevante, motivo de planejamento de ações da OMS (Organização Mundial de Saúde), com metas internacionais a serem alcançadas para a 
promoção da qualidade da assistência à saúde. Portanto, pode ser capaz de contribuir para a reflexão quanto as realidades vividas e suscitar melhorias da assistência nas instituições de saúde.

\section{Metodologia}

Trata-se do tipo de estudo exploratório-descritivo de natureza qualitativa. A pesquisa foi realizada em um hospital público, de grande porte, localizado num município do Sudoeste da Bahia. A população do estudo foi composta por enfermeiros que trabalham no referido hospital, cuja amostra foi formada por profissionais que atuam nos períodos matutino, vespertino e noturno nas unidades de clínica médica e cirúrgica, perfazendo o total de 12 participantes. O fechamento amostral ocorreu no momento em que houve uma saturação teórica emitida nas falas dos informantes, ou seja, no momento em que os dados apresentarem certa repetição (FONTANELLA; RICAS; TURATO 2008). Foram adotados os seguintes critérios de inclusão: enfermeiros que trabalham nos três turnos, que aceitaram participar voluntariamente da pesquisa e assinaram o Termo de Consentimento Livre e Esclarecido. Foram excluídos aqueles que não aceitaram participar da pesquisa, não assinaram o TCLE ou estavam de licença ou afastados do serviço no período da coleta de dados.

A pesquisa foi realizada no período de 15 a 30 de agosto de 2017 através de entrevista gravada, utilizando-se um roteiro semiestruturado, realizadas individualmente no hospital em horário previamente determinado junto aos participantes.

As entrevistas, após serem realizadas, foram transcritas para o programa Word/Starter 2010. Os dados foram analisados visando à identificação das categorias e unidades temáticas a partir da utilização do referencial de Bardin. As análises dos dados foram operacionalizadas a partir das seguintes ações: primeiramente foi realizada a leitura flutuante e aprofunda dos dados grifando os relatos significativos, semelhantes e diferentes; recortes de fragmentos grifados; busca de categorias a partir das temáticas mencionadas no objetivo; elaboração de uma lista de fala dos entrevistados por categorias; escolha das falas que possuem maior poder de síntese e abrangência, informações dentre os conteúdos das entrevistas para exemplificar as categorias emergentes. A análise foi ancorada no referencial teórico escolhido. Para garantir a privacidade de cada participante, a entrevista foi codificada de E1 a E12, correspondendo $\mathrm{E} 1$ à primeira entrevista $\mathrm{e}$, assim, sucessivamente.

As informações foram levantadas após a aprovação do CEP da Faculdade Independente do Nordeste (FAINOR), CAAE 69868917.9.0000.5578, seguindo-se a assinatura do Termo de Consentimento Livre e Esclarecido (TCLE) pelos que se dispuseram a participar da pesquisa, 
atendendo, portanto, o que preconiza a Resolução 466/12 referente a pesquisas com seres humanos, resguardando-os com relação à autonomia, sigilo e anonimato.

\section{Resultados e Discussões}

Os resultados sobre o perfil dos participantes apontaram para a faixa etária de 27 a 46 anos; idades entre 32 e 54 anos; apenas 1 sujeito do sexo masculino; tempo de formação variando de 4 a 24 anos e o tempo de trabalho de 10 meses a 24 anos.

Após análise dos dados emergiram três categorias: 1 - Definindo Segurança do Paciente; 2 Erros percebidos na Unidade, e 3 - Estratégias de prevenção de riscos.

\section{Definindo segurança do paciente}

Nas entrevistas, quando questionados "o que você entende por segurança do paciente?", os profissionais demonstraram conhecimento acerca do conceito segurança do paciente, abrangendo a prevenção de eventos adversos e danos à saúde do paciente, como é possível verificar nas falas abaixo:

Segurança do paciente, eu acho que acima de tudo é o profissional, é ter o máximo de atenção com relação a todo procedimento que vai fazer, inclusive, $e$ principalmente medicamento, pra garantir a segurança desse paciente, mais também, existem os outros fatores. Como é.... você ter que usar todos os equipamentos necessários pra que você não leve para esse paciente uma infecção, eu acho que basicamente, isso daí. (E4)

É todo mecanismo, ações, é.... equipamentos, estrutura pra promover uma.... Qualidade na assistência do paciente evitando riscos, danos maiores, danos emocionais, danos físicos, danos medicamentosos, danos patológicos, qualquer tipo de dano para o paciente. (E5)

Tudo que seja necessário para a segurança do paciente, incluindo normas, rotinas, protocolos para que sejam seguidas pela equipe multiprofissional. (E7)

Entendo que a segurança é a gente prestar uma assistência com, é.... a certeza, de o que a gente tá fazendo é o que faz bem ao paciente sem, sem negligencia, sem imperícia, de forma segura para que não cause nem um dano a ele. (E8)

Bom pra mim segurança do paciente é minimizar possibilidades de erros dentro do hospital. (E10) 
Segundo a Classificação Internacional da Segurança do Paciente (CISP), da Organização Mundial de Saúde (OMS), o conceito segurança do paciente significa minimizar o risco a um parâmetro aceitável, reduzindo danos à saúde dos pacientes nas unidades de saúde (WHO, 2009). A fala dos entrevistados E8 e E10, trazem um conceito bem próximo ao da OMS, quando dizem que deve-se minimizar as possibilidades de erros durante a assistência em saúde. Além de minimizar os riscos durante a assistência, a segurança do paciente engloba dimensões do cuidado extremamente relevantes, que devem ser observadas e seguidas, mencionadas pelos participantes:

Bom segurança do paciente, acho que é em primeiro lugar é você zelar pelo bemestar né, são práticas seguras de administração de medicamentos, de assistência, transporte, realização de exames, de procedimentos todo esse cuidado que nós temos que ter de forma holística com... E zelar pela saúde do paciente de forma segura e integral né. (E1)

É... pra mim a segurança do paciente engloba, tanto a segurança física, como psicológica, como social, a gente tem que guardar o nosso paciente de todas as formas, é... de forma mecânica, leito, o quarto em geral né? Quem tá cuidando, quem frequenta, visita e os cuidados próprios da enfermagem contam também, principalmente pra promover essa segurança. Na hora do medicamento, na hora do banho, é na hora do questionamento, na hora da escuta, em todos os momentos. (E12)

Segundo o Programa Nacional de Segurança do Paciente (PNSP), dentre os seis atributos para a qualidade do cuidado, a Segurança do Paciente deve ser considerada sempre primordial, a fim de evitar erros e danos decorrentes da assistência. $\mathrm{O}$ atributo "cuidado centrado no paciente", é extremamente importante com vistas a prestar um cuidado respeitoso, solícito às preferências, às necessidades e aos valores inerentes de cada paciente. Cabe ainda pontuar que a Segurança do paciente tem sido considerada de grande importância por parte dos familiares, dos próprios pacientes e dos diversos gestores da saúde (BRASIL, 2014).

Assim, é de extrema importância que os profissionais de saúde, de todas as categorias, ponderem a segurança do paciente como uma ferramenta potente, capaz de mudar e revolucionar a maneira como as unidades de saúde prestam serviços aos seus usuários. Através de métodos e conhecimentos que visam oferecer um sistema de saúde correto, confiável e que minimize a incidência de erros ou danos à saúde do paciente, é possível aumentar a qualidade do cuidado prestado (BRASIL, 2014). 


\section{Erros percebidos na unidade}

Ao serem questionadas quanto aos erros mais frequentes no ambiente de trabalho, foram muito citados a falta de identificação do paciente e erros de administração de medicamentos. Sabe-se que os erros de medicações são os que mais ocorrem na prática assistencial, estes foram apontados como os erros mais graves pelos entrevistados:

Não identificação com pulseiras; Mudanças de decúbito não comprida; Altas precoce. (E7)

Entre os erros, envolvidos desde alguns erros de prescrição que isso vai interferir também na administração do medicamento. Isso também não engloba somente aspectos da enfermagem, os erros mais também de outros profissionais que envolve a equipe multidisciplinar. Aí muitos erros estão em cadeia. Um vai levando o outro a errar também. Prescreve uma medicação de forma errada, aí faz aquela medicação de forma errada e isso leva a, né um, algum, problema, ou alguma "iatrogênia". (E2)

A fala do profissional (E6), mostra a repercussão que os erros relacionados à medicação podem gerar, especialmente na administração errada, na vida de um paciente e a divulgação pela mídia:

\footnotetext{
Bem... E.... os erros que podem acontecer graças a Deus aqui no ambiente de trabalho, meu ambiente de trabalho a gente tenta, é....evitar o máximo, né? Erros, o que eu acho que em geral pode acontecer não no теи como em qualquer um da área da saúde é infelizmente por falta de atenção, na verdade as vezes até por negligencia, mesmo, é.... uma troca de medicação. Entendeu? É.... uma ... administração, uma, uma via é.... errada, já aconteceu de medicação que era pra ser administrada ne sonda nasogástrica, ser administrada em acesso central, entendeu? Então assim, acaba que são erros graves, né? São erros que j.... custam, né as vezes a depender do, a depender da gravidade, a vida do paciente, então basicamente em relação a segurança do paciente eu acho que os erros que mais são, é que mais acontecem nas unidades de internação, que volta e meia tá ai na mídia é em relação a administração de medicação. (E6)
}

Inegavelmente o cuidado em saúde traz diversos benefícios para a evolução dos pacientes, no entanto, apesar das diversas evidências positivas do cuidado, o erro em saúde tem ocorrido com mais frequência e gravidade nos últimos anos (BUENO; FASSARELA, 2012). Entende-se por erro em saúde, qualquer evento adverso ocorrido durante a assistência em saúde, que venha a causar algum dano para o paciente. Estes eventos são, culturalmente, tratados com caráter punitivo.

Os profissionais de enfermagem são os principais responsáveis pela minimização dos riscos e pela Segurança do Paciente, especialmente quando refere-se ao gerenciamento de risco do paciente, tanto pelo quantitativo de profissionais quanto pelo cuidado que é prestado ao paciente em 24 horas. 
Apesar de pouco conhecida, no Brasil existe desde 2005 a Rede Brasileira de Enfermagem e Segurança do Paciente (REBRAENSP), que foi criada no sentido de orientar profissionais acerca da relevância da Segurança do Paciente e também da mudança de cultura das instituições hospitalares, com o intuito de incentivar a implementação da cultura de segurança (CALDANA et al, 2015). As instituições de saúde estão buscando, gradativamente, implementar a cultura de segurança que constitui um dos requisitos essenciais para prevenir a ocorrência de danos, incentivando a comunicação dos erros sem o caráter punitivo e sim com vistas a discutir soluções para evitar a repetição do erro (BRANQUINHO; SILVA, 2014).

O Ministério da Saúde, através da Agencia Nacional de Vigilância Sanitária (ANVISA), instituiu o Programa Nacional de Segurança do Paciente (PNSP) que estabeleceu os Protocolos Básicos de Segurança do Paciente, como diretrizes para as instituições de saúde, a fim de proporcionar a segurança e qualidade do cuidado ofertado ao paciente. Os Protocolos vão orientar os profissionais na ampliação da segurança por serem instrumentos sistêmicos para construir uma prática assistencial segura (BRASIL,2014).

Alguns entrevistados deram ênfase aos erros de medicação causados pela identificação do paciente através do número de leito e não do seu nome; pelo dimensionamento inadequado de pessoal; troca de pacientes e o número insuficiente de bombas de infusão para determinados tipos de medicamentos:

No nosso trabalho, é.... a gente vê o paciente não como um leito, sabe? Leito A, leito dois A, leito três A, eu acho que a gente tem que ver o paciente como, o nome do paciente, é seu Aurino, é Dona Maria, É Dona Joana, é... porque o erro pode acontecer exatamente nesse momento, cê ta definindo como um leito, como um número, e de repente você ta fazendo algum medicamento errado num paciente que num era pra, pra ter sido feito. (E4)

Podem acontecer em termos de erros justamente por causa desse volume, essa quantidade distribuição de pessoal que não é equivalente ao número de funcionários, né? Pode acabar alguns erros de administração de medicação, talvez alguma dose, a dose inadequada, a via, né? As vezes pode acontecer também de uma troca de mediação, né? São coisas que podem acontecer por conta disso. (E8)

[...] Erros de comunicação de medicação, tipo, é.... o mesmo paciente com o mesmo nome, na mesma enfermaria, então você pode está levando um erro de.... Administração de medicação [...] É isso mesmo, que as vezes a gente coloca o paciente no mesmo leito, na mesma enfermaria, com o mesmo nome e aí a gente sempre tem que tá trocando, não pode colocar o mesmo nome do paciente, esse, esse período agora a gente teve dois Marcelos, ai a gente trocou esses dias pra não complicar até mesmo pras meninas não poder mudar as medicações nas gazelas, o nome mesmo é o maior dos riscos que a gente tem aqui. (E9) 
Bom.... é.... aqui a gente ver, pelo menos assim, eu consigo perceber poucos, mais o que eu já presenciei medicação errada, em via errada, é.... acho que os mais foram esses mesmos, medicação no paciente errado e a via da medicação errada. (E10)

E uma outra coisa é a questão também da, da bomba de, de infusão para algumas medicações que a gente não tem na quantidade suficiente pra atender o paciente aqui na clínica, então alguns usa, outros não, e ai fica difícil de fazer o controle, por que, tem medicação que é estabelecida né o tempo, de uso e que o gotejamento, com o gotejamento não fica muito correto. (E11)

O erro de medicação pode estar relacionado a diversos aspectos como: "prática profissional, ao produto usado, ao procedimento, à má comunicação na prescrição, aos rótulos, às embalagens, à preparação, à dispensação, à distribuição, ao monitoramento, e ser cometido por qualquer membro da equipe, em qualquer momento do processo do cuidado" (LOPES et al, 2012, p.96).

Estudos realizados no Brasil mostram que existem ainda diversos erros relacionados à administração de medicamentos. Uma pesquisa realizada em Minas Gerais, no ano de 2012, em um Centro de Terapia Intensiva (CTI), avaliou fatores inerentes aos erros durante preparo e administração de medicamentos, pela equipe de enfermagem. Os autores constataram que de 136 fármacos administrados, em 43 ocorreram erros relacionados à: via e dose, diluição, omissão, horário da medicação e velocidade de infusão do fármaco. Ainda foi evidenciado que as causas dos erros guardavam forte relação com as diversas tarefas desenvolvidas pelo mesmo profissional e as mudanças não padronizadas de prescrições médicas (LEMOS; SILVA; MARTINEZ, 2012).

Dentre as medidas adotadas para a prevenção de erros de medicação utiliza-se os "certos da medicação" que se constitui um favorável instrumento para este fim, uma vez que minimiza absurdamente a possibilidade de erro. Segundo o MS, existiam apenas cinco certos da medicação: 1 . Medicação certa; 2. Paciente certo; 3 . Dose certa; 4. Via certa; 5. Horário certo; alguns anos mais tarde foram incorporados mais dois certos: 6 . Registro certo; 7 . Ação certa; a fim de minimizar ainda mais a ocorrência de erros e eventos adversos, hoje, foram adicionados mais dois certos: 8 . Forma farmacêutica certa; 9. Monitoramento certo (BRASIL, 2013).

Conforme discutido anteriormente, entre os protocolos básicos de segurança do PNSP destacase aquele que versa sobre a segurança na prescrição, uso e administração de medicamentos, a ser aplicado em todos as instituições que proporcionam cuidados à saúde, em todos os níveis de complexidade, para o uso de medicamentos para qualquer finalidade que seja.

Os erros foram apontados como sendo resultado, entre outras coisas, da excessiva carga de trabalho; da insuficiência de recursos humanos, materiais ou insumos para a prestação do cuidado, como evidenciado nas falas: 
Acho que é, erros de manipulação porque as vezes a equipe não tem noção do quadro clinico do paciente pela quantidade excessiva de trabalho, excesso de pacientes as vezes não tem tempo de ta olhando prontuário_de ver qual o diagnostico que o paciente tem, quais são os cuidados ... dia a dia... cê vai cortar? (E1)

As dificuldades que a gente tem além da falta de entendimento as vezes da equipe porque não tem um treinamento adequado em relação a isso e a falta de pessoal por exemplo também é um problema. [...], as necessidades não nos permite dar uma assistência $100 \%$ pra evitar os, os, riscos, né... da internação. (E3)

Eu acho que a gente começa a falhar por a demanda que a gente tem, maior que o número de funcionários, isso acaba resultando em alguns erros [...]. (E8)

É, tem erros de toda natureza, é... o.... começar pela estrutura física, higienização, que não é da melhor qualidade como deveria ser, quantidades de funcionários que é pequena, a necessidade de ter uma acompanhante que também não é treinado, é.... a parte física, os, até suporte de soro que as vezes ta quebrado quase caindo, mais a gente precisa utilizar, cama quebrada, torta que não levanta a cabeceira, as vezes não tem a grade[...]. (E 12)

Ressalta-se que o PNSP, também pontua que a pressão sobre os funcionários, as superlotações, as exigências de altas produtividades fazem com que os eventos adversos ocorram com mais frequência. Um estudo realizado em 2012, traz que os principais fatores da ocorrência de erros eram: dimensionamento inadequado de pessoal e as superlotações das instituições (FASSINI; HAHN, 2012).

Em 2014 foi realizado um estudo num Hospital do Piauí, que mostrou elevados índices de erros de medicação. Os erros constatados ocorreram pela não observância das regras de biossegurança; com 40\%; administração do medicamento na hora errada, com quase $38 \%$ além da diluição realizada de forma errada em 35,6\%. Em relação aos erros no momento da administração, observou-se: não cumprimento das normas de biossegurança, administração do medicamento no horário errado, omissão de dose terapêutica, e mais uma vez velocidade de infusão equivocada (DE GALIZA et al, 2014).

Uma pesquisa realizada pela Universidade Tiradentes, em 2013, mostrou que os erros relacionados à medicações ocorriam principalmente devido a sobrecarga de trabalho, a dificuldade de entendimento algumas prescrições médicas ilegíveis e faltas de embasamento técnico/científico (DOS SANTOS et al, 2014).

É extremamente importante que o enfermeiro ao ser supervisor, ou assistencial, tenha o aporte teórico e prático adequado, na administração de medicamentos no que diz respeito a farmacocinética e farmacodinâmica, reações adversas, vias de administração e monitorização. Além disso, que lance mão de protocolos ou incentive a equipe à utilizá-los pois a falta ou a deficiência destes conhecimentos, resulta em falhas ou erros que poderiam ser evitados (DOS SANTOS et al, 2014). 


\section{Estratégias de prevenção de riscos}

Quando questionados acerca das estratégias para a prevenção de riscos, os profissionais relataram estratégias muito particulares de cada um, demostrando pouco conhecimento acerca dos protocolos e dos passos globais para segurança do paciente:

[...] as estratégias é tá sempre em alerta né, a gente pede sempre a equipe a está em alerta, um promove um apoio ao outro na dúvida, no discutir uma tensa medicação, [...] principalmente os riscos de queda né a gente sempre está em alerta para cuidar do paciente, a grade, o uso da grade em pacientes que estão com risco queda, problemas neurológicos especialmente, hipotensão em alguns casos, mais sempre está um apoiando o outro pra prevenir justamente esse risco de, ao paciente. (E2)

[...] a gente como enfermeiro do setor, é tá sempre falando com o técnico de enfermagem da importância de, de você tá, é, é, olhando esse paciente dessa forma que eu te falei anteriormente, agora eu acho que falta, se a gente for falar, falta um pouco também se a gente for falar de uma capacitação ou tá sempre tendo, é, é educação continuada pra que sempre esteja reforçando a prevenção desse, desse para que esses erros não aconteça com a prevenção dos riscos de uma infecção ou, no ambiente hospitalar. (E4)

Eu acredito que a estratégia fundamental seria uma.... a.... capacitação permanente desses profissionais que trabalham com esse paciente, é claro que quando a gente passa, também em infraestrutura em equipamentos adequados né, seria o fato de você, verificar, quais são os [...]. (E5)

As estratégias são, é.... basicamente você adotar aqueles passos, né.... Aqueles passos que a gente ver na, que a gente conhece na teoria [...] perguntar nome do paciente, é.... perguntar nome e sobrenome do paciente pra evitar uma, uma medicação equivocada [...] focando aqui em relação as estratégias do, adotadas pra promover a prevenção de risco, é isso, é você adotar a, os passos de segurança mesmo, entendeu? Evitar o máximo que esse paciente sobra qualquer risco e possa ser promovido por uma negligencia da parte do profissional, né? Que inclui mesmo ter total segurança do que você tá fazendo, preparar a medicação [...] Prestar muita atenção, no rotulo, ler o rotulo, não é uma coisa boa é uma coisa que é necessário. [...].(E6)

Falando individualmente né por que isso é um problema de estrutura que eu citei anterior, mais individualmente o que a gente tenta fazer é manter a concentração na hora dos cuidados prestados, conferindo aquele, né? Os cincos certos, que já passou pra nove, que já passou pra dose, que já, que nem sei quanto tá agora. Né? A certeza da administração, conferindo nome, medicação, via, paciente, essas coisas dá uma ...certa segurança pra a gente tá corrigindo esses possíveis erros. (E8)

Os participantes revelaram desconhecimento dos protocolos, pois poucos citaram intervenções concretas neste sentido. Percebe-se, nos depoimentos, a necessidade de capacitação e educação permanente. 
O atual conceito de segurança do paciente traz como principais responsáveis pela ocorrência de erros as falhas e deficiências no sistema, com a premissa de que não se deve responsabilizar exclusivamente o profissional. Mesmo havendo a concepção de que o ser humano comete erros, a segurança e a eficiência do cuidado ofertado ao paciente depende da organização do serviço e todos os processos envolvido no plano terapêutico daquele indivíduo que envolvem momentos desde a admissão até alta do paciente (DOS SANTOS et al, 2014).

Na busca pela prevenção de danos e riscos ao paciente, diversos órgãos ao redor de todo o mundo desenvolveram estratégias que auxiliam na segurança do paciente no âmbito hospitalar. Dentre os órgãos cita-se a o Ministério da saúde, que desenvolveu o PSNP e a Joint Commission on Acreditation of Healthcare Organizations (JCAHO), órgão de acreditação hospitalar que traz basicamente seis metas a serem cumpridas pelas instituições de saúde: identificar os pacientes corretamente; melhorar a comunicação; melhorar a segurança com medicamentos de risco; eliminar cirurgias em membros ou pacientes errados; reduzir o risco de adquirir infecções e reduzir o risco de lesões decorrentes de quedas do leito (DE SOUZA; DA SILVA, 2014).

A REBRAENSP, órgão brasileiro de Segurança do Paciente, junto com o COREN, elaboraram dez passos, baseados em evidências científicas atualizadas, para subsidiar profissionais durante a prática, sendo eles: Identificação do paciente; Cuidado limpo e cuidado seguro - higienização das mãos; Cateteres e sondas - conexões corretas; Cirurgia segura; Sangue e hemocomponentes - administração segura; Paciente envolvido com sua própria segurança; Comunicação efetiva; Prevenção de queda; Prevenção de úlcera por pressão e Segurança na utilização de tecnologia (CALDANA et al, 2015).

Quando questionados se existem educações continuadas e qual a frequência, as falas dos profissionais foram unanimes quanto a carência de treinamentos, como é possível visualizar abaixo:

Bom, educação continuada a gente não tem o que a gente faz é sempre uma
conscientização da equipe, eu mesmo como relatei o erro que eu percebo, que eu
percebi já nesse tempo foi a questão da medicação em via errada, então o quê que a
gente fez? Eu chamei a, a pessoa em loco pra poder resolver essa situação, acabei
não expondo só mesmo na questão de educar e conscientizar. [...] É o que a gente faz
$e ́$ realmente essa conscientização dos técnicos de enfermagem em relação a
medicaçôes a fazer o uso de dez certos da medicação. E por aí vai. (E10) Às vezes sim, não tem assim uma frequência por exemplo de meses, dias... é muito relativo é quando a coordenação do hospital oferece esse treinamento mesmo. Mais não tem uma periodicidade de treinamento não. (E1)

Nunca. Tudo que eu sei, nunca dependeu do.... Você? Eu estou sendo entrevistado aqui no hospital público onde eu trabalho já trabalhei em várias instituições inclusive particulares onde a gente era constantemente treinado pra isso, treinado e observado, supervisionado mais aqui infelizmente hoje a gente tem uma evolução de enfermagem que sinaliza algumas coisas importantes porem treinamento mesmo eu já tenho 10 anos de estado e eu nunca tive um treinamento aqui. (E3) 
É perceptível, através das falas, a necessidade de capacitação dos profissionais, haja vista a não realização desta pela instituição. O MS, traz na PSNP, que deve-se incluir o tema segurança do paciente na educação permanente, uma vez que a saúde incorpora tecnologias muito rapidamente, como por exemplo, a inclusão de novos medicamentos em listas padronizadas (BRASIL, 2014). A PSNP ainda orienta que deve existir um programa de educação permanente dos profissionais da Saúde, acerca da Segurança do paciente.

\section{Considerações Finais}

Com base nos dados coletados e nas discussões levantadas acima, foi possível perceber que os participantes apresentaram conhecimentos limitados acerca da temática "Segurança do Paciente", bem como sua aplicabilidade na prática profissional, demostrando a necessidade de capacitações acerca do tema. Foi possível ainda constatar que existem barreiras para que o cuidado prestado ao paciente seja efetivo, livre de riscos ou eventos que venham causar agravos à saúde.

Diante desses resultados, entende-se que medidas urgentes devem ser adotadas para a implementação de uma cultura de segurança do paciente, bem como a efetiva implantação dos protocolos básicos de segurança e o gerenciamento de riscos.

Por fim, ressalta-se como limitação do estudo, a realização da pesquisa apenas com enfermeiros de dois setores, sendo importante que se desenvolvam mais estudos com esta temática, e com outros profissionais da saúde.

\section{Referências}

BRANQUINHO, Nayla Cecília; SILVA, A. E. Cultura de Segurança do paciente em instituições de saúde: um estudo bibliométrico. Enferm Global, v. 33, p. 349-61, 2014.

BRASIL. Ministério da Saúde. Protocolo de segurança na prescrição, uso e administração de medicamentos. Ministério da Saúde e ANVISA em parceria com FIOCRUZ e FHEMIG. Brasília: Ministério da Saúde, 2013. $\quad$ Disponível em: http://portalarquivos.saude.gov.br/images/pdf/2014/julho/03/Protocolo-Medicamentos.pdf 
BRASIL. Ministério da Saúde. Documento de referência para o Programa Nacional de Segurança do Paciente / Ministério da Saúde; Fundação Oswaldo Cruz; Agência Nacional de Vigilância Sanitária (ANVISA). - Brasília: Ministério da Saúde, 2014.

BUENO, A.A.B.; FASSARELLA, C.S. Segurança do Paciente: uma reflexão sobre sua trajetória histórica. Revista Rede de Cuidados em Saúde. v. 6, n. 1, p:1-9, 2012.

CALDANA, G. et al. Rede Brasileira de Enfermagem e Segurança do Paciente: Desafios e Perspectivas. Texto Contexto Enferm, Florianópolis, v.24, n.3, p: 906-11 2015

DE GALIZA, D.D.F.; DE MOURA, O.F.; DE BARROS, V.L.; LUZ, G.O.A. Preparo e administração de medicamentos: erros cometidos pela equipe de enfermagem. Rev. Bras. Farm. Hosp. Serv. Saúde São Paulo v.5 n.2, p:45-50, 2014.

DE SOUZA, R.F.F.; DA SILVA, L.D. Estudo exploratório das iniciativas acerca da segurança do paciente em hospitais do Rio de Janeiro. Rev enferm UERJ, v.22, n.1, p:22-8, 2014.

DIAS, J.D et al. Compreensão de enfermeiros sobre segurança do paciente e erros de medicação. REME Rev Min Enferm. v.18, n.4, p: 866-873, 2014.

DOS SANTOS, D.S. et al. Segurança do paciente: fatores causais de eventos adversos a medicamentos pela equipe de enfermagem. Ciências Biológicas e da Saúde, v. 2, n.2, p. 19-30, 2014.

FASSINI, P.; HAHN, V. Riscos à segurança do paciente em unidade de internação hospitalar: concepções da equipe de enfermagem. Rev Enferm UFSM, v.2, n.2, p:290-299, 2012.

FONTANELLA, B.J.B.; RICAS, J.; TURATO, E.R. Amostragem por saturação em pesquisas qualitativas em saúde: contribuições teóricas. Cad saúde pública. v.24, n.1, p:17-27, 2008.

LEMOS, N.R.F.; DA SILVA, V.R.; MARTINEZ, M.R. Fatores que predispõem à distração da equipe de enfermagem durante o preparo e a administração de medicamentos. rem $E$ - Rev. Min. Enferm., v.16, n.2, p: 201-207, 2012.

LOMBARDI, N.F et al. Analysis of the discrepancies identified during medication reconciliation on patient admission in cardiology units: a descriptive study. Rev. Latino-Am. Enfermagem. V.24:e2760. 2016.

LOPES, D.M.A. et al. Análise da rotulagem de medicamentos semelhantes: potenciais erros de medicação. Rev Assoc Med Bras, v.58, n. 1, p:95-103, 2012.

OLIVEIRA, R.M. et al. Estratégias para promover segurança do paciente: da identificação dos riscos às práticas baseadas em evidências. Esc Anna Nery. v.18, n.1, p:122-129, 2014.

REIS, C.T.; MARTINS, M.; LAGUARDIA, J. A. Segurança do paciente como dimensão da qualidade do cuidado de saúde - um olhar sobre a literatura. Ciência \& Saúde Coletiva, v.18, n.7, p: 2029-2036, 2013.

WORLD HEALTH ORGANIZATION (WHO). World Alliance for Patient Safety. Global Patient Safety Challenge: 2005-2006. Genova: WHO; 2005. 
World Alliance for Patient Safety, Taxonomy: The Conceptual Framework for the International Classification for Patient Safety: final technical report. Genebra; 2009.

\section{Como citar este artigo (Formato ABNT):}

SANTOS, Laricy S dos; ARAÚJO, Giovana F. Nível de Informação da Equipe de Enfermagem sobre Segurança do Paciente num Hospital Público. Id on Line Revista Multidisciplinar e de Psicologia, 2017, vol.11, n.38, p. 322-335. ISSN: 1981-1179.

Recebido: 28.10 .2017

Aceito: 30.10 .2017 